\title{
Experimental Investigation of Flow Structure Formation in a Heated Duct Flow
}

\author{
Tobias Mey, Andreas Westhoff and Claus Wagner \\ Institut für Aerodynamik und Strömungstechnik des \\ Deutschen Zentrums für Luft- und Raumfahrt e.V. Göttingen \\ Bunsentraße 10, Göttingen, D-37075 \\ tobias.mey@llg-ev.de, andreas.westhoff@dlr.de, claus.wagner@dlr.de
}

\begin{abstract}
The pattern formation of a Poiseuille flow in a rectangular duct, which is heated from below, is studied experimentally for the Reynolds number $\operatorname{Re}=200$ within the Rayleigh number range $4 \times 10^{8} \leq \mathrm{Ra} \leq 7 \times 10^{8}$. The channel has an aspect ratio of $\Gamma_{y z}=$ width $:$ height $=25: 2$. As working fluid, water $(\operatorname{Pr} \approx 6)$ is used. In order to study the influence of buoyancy on the forced flow, velocity fields are measured plane parallel to the heated bottom plate by means of Particle Image Velocimetry (PIV). Furthermore, the measured data is analyzed concerning the flow structure formation as a function of the temperature difference between the inflow and the bottom of the duct. In this connection, two different mechanisms can be distinguished, which cause the transition from a laminar to a turbulent flow: a successively progressing transition on the one hand and, above a certain Archimedes number $\mathrm{Ar}$, an abrupt transition.
\end{abstract}

\section{Introduction}

The term convection refers to a mechanism of thermal energy propagation, at which heat is transported in the form of internal energy of atoms or molecules. If the heat transport is driven by a flow induced by a pressure gradient, one talks about forced convection. A purely buoyancy induced flow is called free convection. The superposition of both types of convection is denoted as mixed convection.

Convective flows are subject of the ongoing research since decades and have a fundamental relevance in science, technology and nature. Such as in those flows found in the oceans [11], in the earth's atmosphere [8] or in the magma of the earth's mantle [9] as well as the granular surface of the sun, which arises by reason of raising and dropping elements of fluid [1]. Mixed convection is of elementary importance in geothermal and solar thermal energy or in heat exchanger [7]. Particularly the formation of flow patterns, which mostly determine the transport of heat, is of great importance and is related to many unanswered questions. 
Therefore this study focuses on the experimental investigation of mixed convection in a rectangular duct, which is heated from below. Thereby a Poisseuille flow, induced by a pressure gradient, is superimposed by a buoyancy induced secondary flow. Critical parameters are the aspect ratio of the channel $\Gamma_{y z}=W / H$, the Reynolds number $\operatorname{Re}=\frac{u_{0} H}{v}$ as a measure of forced convection, the Rayleigh number $\mathrm{Ra}=\frac{g \beta \Delta T H^{3}}{\alpha \nu}$ as a measure of free convection as well as the Prandtl number $\operatorname{Pr}=\frac{v}{\alpha}$ as a fluid specific property ${ }^{1}$. In the following, instead of the Rayleigh number, we will specify the Archimedes number $\operatorname{Ar}=\operatorname{Ra} /\left(\operatorname{Pr} \times \operatorname{Re}^{2}\right)$, which is the global ratio of buoyancy to inertia forces.

In a study referring to the elucidated problem formulation, Chiu and Rosenberger $[5,6]$ identify two different flow regimes. At the very beginning of the duct, the flow is dominated by forced convection. Further downstream buoyancy forces reveal a significant influence as a result of the temperature difference between the fluid and the heated bottom of the duct. In the given geometry and for the chosen flow parameters $(\operatorname{Re}=44.8, \operatorname{Pr}=0.71, \mathrm{Ar}=3.4)$, ten stationary convection rolls develop, which are oriented in flow direction. As a result, the thermally driven flow $w$ modulates the forced flow $u$ as schematically shown in figure 1 .

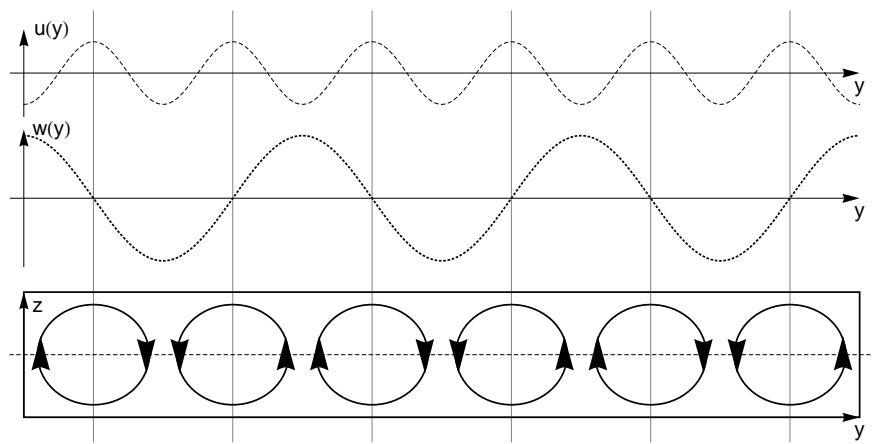

Fig. 1 Velocity profiles $u(y)$ and $w(y)$ and a schematic representation of the thermally influenced duct flow as a cross section view

In an additional study, Chang et al. $[4,3]$ report on the influence of the aspect ratio $\Gamma_{y z}=W / H$ on the flow. They show, that raising $\Gamma_{y z}$ (= broadening the channel) in the range $2<\Gamma<12$ makes a laminar flow become turbulent, while all other parameters are kept constant. They observe the same behavior in case of reducing the Reynolds number. The reason of this behavior is given by the rising role of buoyancy forces $(\propto \mathrm{Ra}=$ const) toward inertia forces $(\propto \mathrm{Re})$.

A detailed investigation of the process, how stationary roll structures in the flow

${ }^{1} \mathrm{~g}$ : gravitational acceleration, $H$ : height of the duct, $\Delta T$ : temperature difference between inflow and bottom of the duct, $u_{0}$ : mean flow velocity in $x$-direction, $W$ : width of the duct, $\alpha$ : thermal diffusivity, $\beta$ : volumetric thermal expansion coefficient, $v$ : kinematic viscosity, $\rho$ : density 
evolve, has been published by Benderradji et al. [2]. Employing both experimental and numerical methods, they find two different kinds of mechanisms. One they call the "lateral wall effect", whereby pairs of convection rolls successively form, starting at the side walls progressing to the channel's centerline. Above a critical Archimedes number of $\operatorname{Ar} \approx 2.6$ the formation of all rolls occurs simultaneously over the whole channel width.

In the present article we examine the development of a third, instationary flow regime, which is understood merely insufficiently yet. Based on the PIV measurement data we temporally analyze the velocity field for different Archimedes numbers. In this connection, depending on Ar, we define areas in the measuring range with a stationary flow structure or an instationary one respectively. Thereby we find the same mechanisms in the transition from a laminar to a turbulent flow as Benderradji et al. describe for the incipient initiation of the convection rolls.

\section{Experimental set-up and measuring technique}

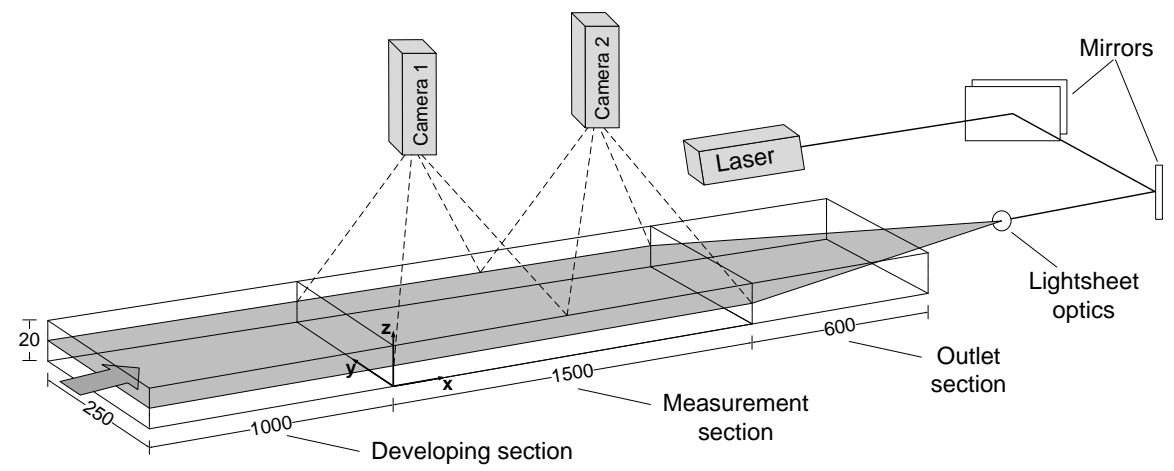

Fig. 2 Perspective view of the experimental set-up including the arrangement of the PIV equipment (not true to scale)

A sketch of the experimental set-up is shown in figure 2. The rectangular duct has a width of $W=250 \mathrm{~mm}$, the height $H=20 \mathrm{~mm}$ and its total length reaches $L=3100 \mathrm{~mm}$. In flow direction it is divided into the three sections: developing section, measurement section and outlet section. The developing section with a length of $L_{\mathrm{DS}}=1000 \mathrm{~mm}$ is sufficiently long to ensure a fully developed laminar flow profile at its tail for flows up to a Reynolds number of $\operatorname{Re}=830$ [10]. It is connected to the measurement section, which has a length of $L_{\mathrm{MS}}=1500 \mathrm{~mm}$ and is heated from below. At the end, an outlet section with $L_{\mathrm{OS}}=600 \mathrm{~mm}$ is located. It should prevent disturbing effects from the test section, which may be caused by the overflow over 
the edge at the end of the duct.

To benefit nearly adiabatic boundary conditions, during the experiment the mean temperature of the forced flow $T_{0}$ is always adjusted equal to the mean room temperature $T_{\text {room }}$. For the isothermal test measurement, the temperature of the heat plate $T_{\mathrm{HP}}$ is set to $T_{0}$ in order to reach $\Delta T=T_{\mathrm{HP}}-T_{0}=0$. For the thermal measurements, $T_{\mathrm{HP}}$ is adjusted accordingly to the listed Archimedes numbers (see table 1).

Instantaneous velocity fields have been measured by means of PIV. As seeding material a titanium dioxide containing polyamide powder with a mean grain size of $20 \mu \mathrm{m}$ and the density $\rho=1.23 \mathrm{~g} / \mathrm{cm}^{3}$ is used. The particles are illuminated with an Nd-YAG-Laser in a plane at $z=0.5 \times H$ plane parallel to the ground of the channel. To this purpose the laser beam is widened and coupled into the duct through its end (see figure 2). Two CCD-Cameras, mounted perpendicularly to the measurement plane, provide double pictures with a resolution of $4000 \times 1400 \mathrm{px}$ at a repetition rate of $f=0.6 \mathrm{~Hz} .1000$ instantaneous velocity fields are determined for the isothermal measurement, 2000 for the thermal cases.

Due to the channel's geometry, there emerge limitations and problems for determining the velocity fields by means of PIV which decrease the quality of the measurement results. Despite all the care taken, the same quality could not be attained throughout the whole channel. Especially in the range $x<300 \mathrm{~mm}$ the power density of the light sheet is too poor to illuminate the seeding particles sufficiently due to its continuously increasing widening. Here an evaluation is not applicable. Hence this region is not considered in the following discussion.

\section{Measured flow structures}

In the following section the results of flow structure formation as a function of $\mathrm{Ar}$ are presented and discussed. The characteristic numbers and the relevant physical parameters are given in table 1.

\begin{tabular}{|l|c|r|l|}
\hline \multicolumn{1}{|c|}{$\operatorname{Ar}[1]$} & $\operatorname{Re}[1]$ & $u_{0}\left[\mathrm{~ms}^{-1}\right]$ & \multicolumn{1}{|c|}{$\Delta T[K]$} \\
\hline $0.00 \pm 0.01$ & $207 \pm 2$ & $9.59 \pm 0.08$ & $0.04 \pm 0.02$ \\
$2.2 \pm 0.3$ & $217 \pm 2$ & $9.56 \pm 0.08$ & $3.7 \pm 0.4$ \\
$4.2 \pm 0.5$ & $199 \pm 2$ & $8.57 \pm 0.07$ & $5.7 \pm 0.7$ \\
\hline
\end{tabular}

Table 1 Overview of the dimensionless and crucial quantities of the investigated flow

At this juncture it should be noted that for all considered cases the $v$-component of the flow velocity is negligible small compared to the $u$-component. Therefore, only the $u$-component of the velocity is considered in the following to determine and characterize the flow structure. Moreover, the velocity $u$ is normalized with the mean inflow velocity $u_{0}$. 

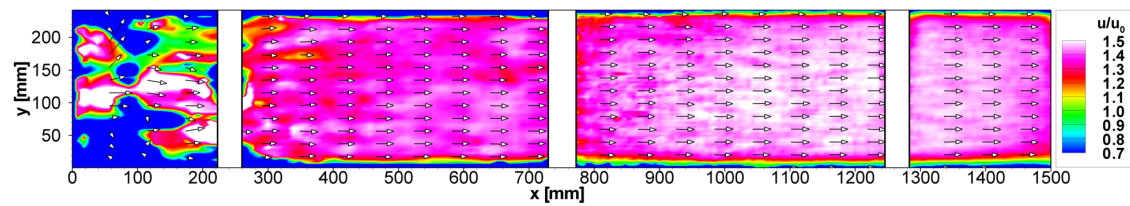

Fig. 3 Contours of the instantaneous velocity field $u(x, y)$ in the measurement plane $z=0.5 \times H$ for $\mathrm{Ar}=0$

Figure 3 shows the instantaneous velocity field for the isothermal measurement $(\mathrm{Ar}=0)$. The three stripes masked out are visually inaccessible areas, where tension belts fix the channel. Depicted is the velocity field in the measurement plane $z=$ $0.5 \times H$. The velocity vectors indicate the flow direction and represented as a contour plot is the scaled velocity $u / u_{0}$.

In line with expectations a homogeneous velocity distribution is found, except for the deviations discussed in section 2 .

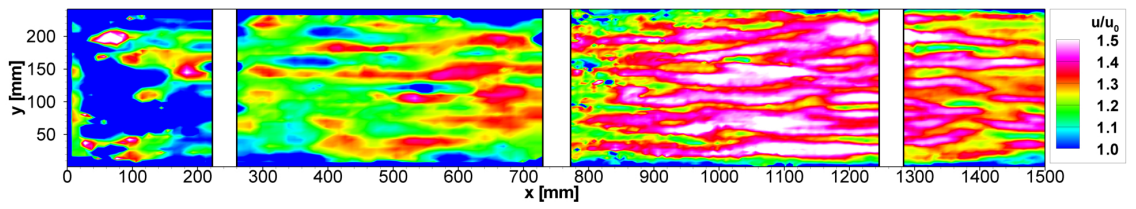

(a) $u(x, y)$ for $\mathrm{Ar}=2.2$
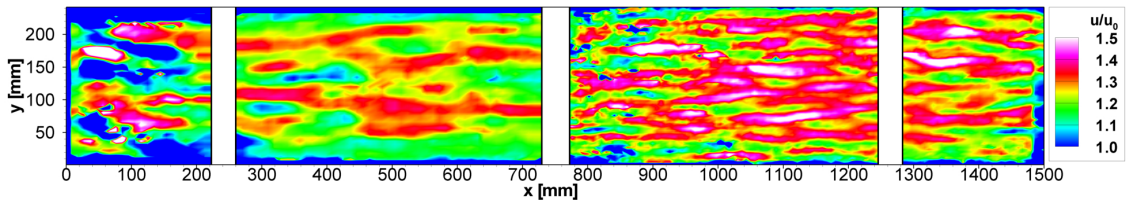

(b) $u(x, y)$ for $\mathrm{Ar}=4.2$

Fig. 4 Contours of the instantaneous velocity fields $u(x, y)$ in the measurement plane $z=0.5 \times H$ for the thermal measurements

Up next we consider the instantaneous velocity fields $u(x, y)$ obtained in the thermal measurements, i.e. with $\Delta T \neq 0$ (see figure 4 ). In all cases, streaks with a maximal velocity, oriented in flow direction, can be observed. In the following we identify them as the centers of the convection rolls, as depicted in figure 1. Analyzing these streaks for increasing Ar we find a breakdown of the streaks more and more frequently into smaller and smaller fragments. The disorder seems to grow and there raise increasingly smaller scaled structures.

The reason for this is the growing modulation of the forced flow by the thermally induced buoyant flow. Due to the increased heat transfer into the fluid, the flow tends to develop further convection centers, which raise the disorder in the flow structure.

To investigate particularly the temporal and spatial stability of the thermal convection rolls, an algorithm is developed, which extracts their positions from the 

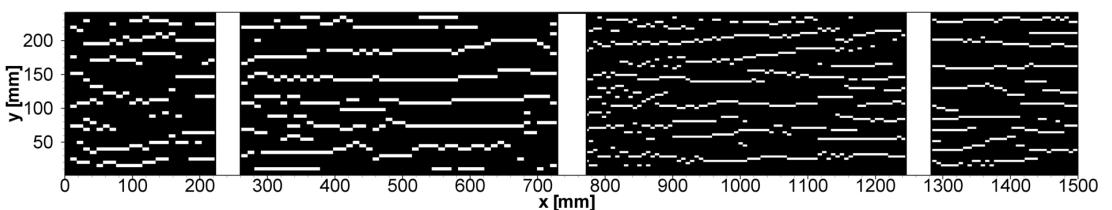

(a) position of rolls extracted from $u(x, y)$ for $\mathrm{Ar}=2.2$

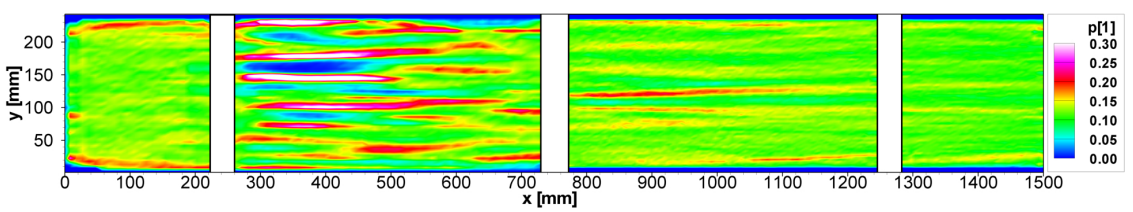

(b) $p(x, y)$ for $\mathrm{Ar}=2.2$

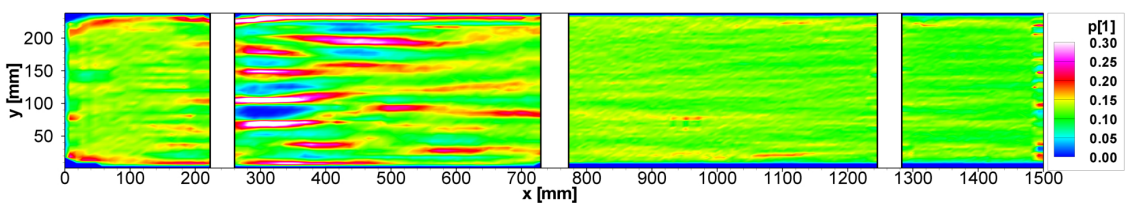

(c) $p(x, y)$ for $\mathrm{Ar}=4.2$

Fig. 5 (a) Example of a binary distribution of the convection rolls' center position, extracted from $u(x, y)$ for $\mathrm{Ar}=2.2$

(b) \& (c) probability distribution of the convection rolls' position $p(x, y)$ for $\operatorname{Ar}=2.2$ and $\operatorname{Ar}=4.2$

velocity field data. For this purpose, at each position $x_{i}$ the velocity distribution $u\left(x=x_{i}, y\right)$ is scanned for local maxima. Each position with a maximum gets the value 1 and each other position is valued with 0 . For one measurement one obtains a spatial binary distribution of the roll's positions, as depicted in figure 5(a). Averaging over 2000 such fields, a probability distribution results, that reveals the positions where convection rolls remain preferentially and which positions they keep clear of.

In figures 5(b) and 5(c) the spatial probability distribution of the thermally induced rolls' position $p(x, y)$ is shown for the measurements at $\operatorname{Ar}=2.2$ and $\operatorname{Ar}=4.2$. Thereby the following conclusions on the spatial as well as the temporal properties of the flow can be made.

In both cases clearly distinct areas with high values $p(x, y)>0.3$ as well as with low values $p(x, y)<0.1$ are found. Summing up adjacent positions of one streak with $p \approx 0.3$, the probability reaches values of up to $p=1$. These areas are interrupted by zones with $p=0$. Corresponding to figure 1 these are exactly the positions with hot fluid raising or cold fluid sinking, respectively.

Following these streaks further downstream, they show quite different lengths comparing between both Archimedes numbers. For $\mathrm{Ar}=2.2$ the longest streak has a length of up to $x=650 \mathrm{~mm}$ and for $\operatorname{Ar}=4.2$ just $x=420 \mathrm{~mm}$ is reached. Thereupon, the streaks end and partially appear again at different positions, but less prominent. Here, the convection rolls loose their fixed positions and begin to vary them tem- 
porally. Thus the ordered structure in the flow decreases and the distribution $p(x, y)$ homogenizes increasingly. As a result, the positions of the rolls are almost equally distributed through the whole width of the channel. Consequently, we find that increasing Ar yields to a more and more early breakdown of the thermal convection rolls.

With the probability distribution shown in figure 5(b) and 5(c) we conclude that for $\mathrm{Ar}=2.2 \mathrm{a}$ uniform distribution for $p(x, y)$ is developed at $x=1200 \mathrm{~mm}$, where for $\operatorname{Ar}=4.2$ it already appears at $x=800 \mathrm{~mm}$.

Considering the reason why and how the ordered structure collapses, two different mechanism can be discerned. In the case of $\mathrm{Ar}=2.2$ the transition takes place successively. First, the convection rolls at the lateral boundaries loose their fixed positions whereupon, after a certain distance, the centrals rolls follow. Thus, we assume that the instability arises from the lateral walls and gradually propagates to the center afterwards. In general we notice that the roll structure most stable occurs exactly in the center of the channel. The reason might be the large velocity gradients at the lateral walls, where the vorticity $\omega(x, y, z)$ is maximal. Hence, these regions tend to an augmented swirling of the flow and become a source of instabilities.

With increasing buoyancy forces this effect disappears. At $\mathrm{Ar}=4.2$, the initially present streaks all end at approximately the same position $x=380 \mathrm{~mm}$. Therefore, a destabilizing mechanism dominates which has an effect through the whole width of the channel. Here, a pure thermal distortion seems to be play a decisive role in opposition to the viscous vortex generation at the measurement with $\operatorname{Ar}=2.2$.

At this junction we note an interesting analogy to the investigation by Benderradji et al. [2], who studied the initial evolution of the roll structure. They found exactly the same mechanisms that occur here at the collapse of the ordered flow structure. They further observed the transition between both mechanism at the critical Archimedes number of $\mathrm{Ar}=2.6$, what is consistent with our observations.

Finally the flow regimes shall spatially be delimited into regions where our results can be interpreted as laminar or as turbulent mixed convection. Based on the representation of the possibility distribution of the convection rolls we can read off easily, where they have clearly favored positions and in which region the ordered structure is lost. Thus, we identify a laminar mixed convection for $x<x_{\text {lam }}$ with $x_{\text {lam }}(\operatorname{Ar}=2.2)=500 \mathrm{~mm}$ and $x_{\text {lam }}(\operatorname{Ar}=4.2)=380 \mathrm{~mm}$, respectively. A turbulent mixed convection can be found in the region $x>x_{\text {turb }}$ with $x_{\text {turb }}(\operatorname{Ar}=2.2)=1200 \mathrm{~mm}$ and $x_{\text {turb }}(\operatorname{Ar}=4.2)=800 \mathrm{~mm}$. The area between both flow regimes is a transition area, where indicators of a laminar as well as of a turbulent flow are found. 


\section{Conclusions}

In the presented report, we observed and analyzed the formation of roll structures in a duct flow heated from below. Therefore the velocity field $u(x, y)$ at $\operatorname{Re} \approx 200$ in the range of $0<\operatorname{Ar}<4.2$ was determined by means of PIV. We found thermally induced roll structures over the whole metrologically accessible length of the test section.

In order to study the stability of the convection rolls, we applied an algorithm to the velocity fields which yields a time averaged probability distribution $p(x, y)$ of the rolls' center positions. By this distribution we identified flow regimes regarded as turbulent and laminar mixed convection. Additionally, we localized a transition region, where laminar as well as turbulent flow has been observed.

Furthermore we determined two different mechanism of the transition from a laminar to a turbulent flow. At $\mathrm{Ar}=2.2$ the regime change proceeds successively, starting from the lateral walls of the channel to its center. This behavior is presumably caused by the viscous vorticity insertion which is maximal in the corners of the duct. In contrast to that, the ordered flow structure breaks down quite abrupt at $\operatorname{Ar}=4.2$. Here, a thermally destabilizing effect dominates, which acts throughout the whole width of the duct in the same extent.

\section{References}

1. Asplund, M.; Nordlund, A.; Trampedach, R.; Allende Prieto, C.; Stein, R. F.: Line formation in solar granulation. Astronomy and Astrophysics 359, 729-742 (2000)

2. Benderradji, A.; Haddad, A.; Taher, R.; Mdale, M.; Abid, C.; Papini, F.: Characterization of Fluid Flow Patterns and Heat Transfer in Horizontal Channel Mixed Convection. Heat Mass Transfer 44, 1465-1476 (2008)

3. Chang, M. Y.; Yu, C. H.; Lin, T. F.: Changes of Longitudinal Vortex Roll Structure in a Mixed Convective Air Flow Through a Horizontal Plane Channel : an Experimental Study. International Journal of Heat and Mass Transfer 40, 347-363 (1997)

4. Chang, M. Y.; Lin, T. F.: Experimental Study of Aspect Ratio Effects on Longitudinal Vortex Flow in Mixed Convection of Air in a Horizontal Rectangular Duct. International Journal of Heat and Mass Transfer 41, 719-733 (1997)

5. Chiu, K. C.; Rosenberger, F.: Mixed Convection Between Horizontal Plates - I. Entrance Effects. Heat Mass Transfer 30, 1645-1654 (1987)

6. Chiu, K. C.; Ouazzani, J.; Rosenberger, F.: Mixed Convection Between Horizontal Plates - II. Fully Developed Flow. Heat Mass Transfer 30, 1655-1662 (1987)

7. Dogan, A.; Sivrioglu, M.; Baskaya, S.: Experimental Investigation of Mixed Convection Heat Transfer in a Rectangular Channel with Discrete Heat Sources at the Top and at the Bottom. International Communications in Heat and Mass Transfer 32, 1244-1252 (2005)

8. Gregory, D.; Rowntree, P. R.: A Mass Flux Convection Scheme with Representation of Cloud Ensemble Characteristics and Stability-Dependent Closure. Monthly Weather Review 118, 1483-1506 (1990)

9. Morgan, W. J.: Convection Plumes in the Lower Mantle. Nature 230, 42-43 (1971)

10. White, F. M.: Viscous Fluid Flow. McGraw-Hill, New York (1974)

11. Webster, P. J.: The Role of Hydrological Processes in Ocean-Atmosphere Interactions. Reviews of Geophysics 32, 427-476 (1994) 